\title{
The Prevalence of Allergic Rhinitis and Associated Risk Factors Among University Students in Anatolia
}

\author{
Kemal Kef iD ${ }^{\prime}$ \\ Selis Güven (iD) ${ }^{2}$ \\ 'Department of ENT, Private Kesan \\ Hospital, Edirne, Turkey; ${ }^{2}$ Department of \\ ENT, Trakya University School of \\ Medicine, Edirne, Turkey
}

This article was published in the following Dove Press journal: Journal of Asthma and Allergy

Introduction: Allergic rhinitis is a common disease in Turkey. However, there are not enough studies on its prevalence. Survey-based studies conducted by experienced and qualified researchers to large populations provide information about the prevalence of allergic rhinitis and risk factors associated with it.

Aim: The aim of this study was to determine the prevalence of allergic rhinitis and related factors in university students in Anatolia.

Methods: An extended and modified form of "The European Community Respiratory Health Survey" was conducted to university students in Turkey. The data were obtained through a face-to-face interview method. $\mathrm{R}$ version 4.0.2 was used for statistical analysis. Data were presented as frequency and percentage. Chi-squared test of independence was used to analyze the relationship between allergic rhinitis diagnosis and other variables. Statistically significant variables were further analyzed with multivariate logistic regression test.

Results: Data was collected from a total of 2020 participants, but 1714 participants were eligible for multivariate analysis. The mean age of the participants was $20.71 \pm 3.12$ years; $42.88(n=735)$ were male, and $57.12 \%(n=979)$ were female. While the rate of those who thought that they had nasal allergies was $23.862 \%(n=409)$, the rate of those diagnosed by a doctor was $15.986 \%(\mathrm{n}=274)$. The most common allergic symptom in the participants was sneezing, and the most common triggering factor was house dust.

Conclusion: We found a high prevalence of $15.986 \%$ doctor diagnosed allergic rhinitis among university students in Anatolia. Genetic, environmental and economic factors were associated with high prevalence of allergic rhinitis.

Keywords: allergic rhinitis, allergy, epidemiology, prevalence, questionnaire, Anatolia

\section{Introduction}

\section{Background/Rationale}

Allergic rhinitis can be described as an inflammatory disease of the nasal mucosa, formed by a reaction mediated by type I immunoglobulin $\mathrm{E}$ ( $\mathrm{IgE}$ ) after encountering the allergen. ${ }^{1}$ It is characterized by nasal symptoms, such as discharge, sneezing, itching, nasal congestion, and itching/redness in the eyes caused by inflammation of the nasal mucosa. ${ }^{2}$

Allergic rhinitis (AR) is one of the most common atopic diseases. ${ }^{3}$ Furthermore, it is the most common type of chronic rhinitis. ${ }^{4}$ Studies have shown that the prevalence of AR varies between $10 \%$ and $58.5 \%$ according to geographical
Correspondence: Kemal Kef Department of ENT, Private Kesan Hospital, Edirne, Turkey

Tel +9053270l 7086

Email dr.kemalkef@gmail.com 
regions. ${ }^{5-7}$ The rate of $\mathrm{AR}$ in adults has been reported at around 21\% in Europe, 25\% in Canada, 27\% in South Korea, and 32\% in the United Arab Emirates. ${ }^{8-11}$ A 2015 study conducted with university students revealed a $58.5 \%$ AR prevalence and the students with AR reported a lower quality of life. ${ }^{7}$

It is stated that the most common allergic disease in Turkey is AR. ${ }^{12}$ The rate of allergies in Turkey is shown in different studies in the range of $7-25 \% .^{12-15}$ However, the frequency of allergies has been reported to vary depending on countries, regions, as well as urban or rural settings. ${ }^{12}$

Although it is a common ailment, a study conducted in the UK found that only $18 \%$ of individuals with rhinitis visited general practitioners for the previous 2 years due to this problem. ${ }^{16}$ Another study in France indicated that $19 \%$ of 230 patients with typical AR symptoms did not consult a doctor for nasal problems. ${ }^{17}$

Allergic rhinitis has economic, clinical, and social negative consequences. It can lead to workday loss in adults and school day loss and learning disabilities in children. $^{18-20}$ In a study conducted by Seedat et $\mathrm{al}^{21}$ $39.4 \%$ of the students suffering from AR reported frequently feeling tired and $19.5 \%$ of the students with AR said they felt miserable because of their nasal symptoms. The quality of life decreases in AR patients, a scientifically significant relationship was found between AR and impairment in social functioning. ${ }^{7}$ If $\mathrm{AR}$ is uncontrolled and untreated, it may lead to secondary diseases (such as conjunctivitis, sinusitis, middle ear infections, jaw and teeth development disorders, and asthma) or delay in the treatment of such existing diseases. ${ }^{22}$

\section{Objectives}

This study aimed to determine the prevalence of AR and related risk factors among University students in Anatolia.

\section{Methods}

\section{Study Design}

A descriptive cross-sectional study design was used. The population of the study was determined as students studying at Trakya University during the study year 2019. The data were obtained through a face-to-face interview method with a team of Trakya University Faculty of Medicine students, using the modified "The European Community Respiratory Health Survey (ECRHS) Questionnaire" with additional questions. ${ }^{23}$ The participants were informed about the content and purpose of the study and asked to respond after signing a consent form.

Ethical approval for this research was received from Trakya University Faculty of Medicine Clinical Research Ethics Committee with the number "TUTF-BAEK 2019/ 188 " and date 22.04.2019. The reporting of the study was done per the STROBE guideline. ${ }^{24}$ The research was carried out in students studying at Trakya University between May 2019 and September 2019. During the study period, Trakya University had approximately 47 thousand students. Participants were identified by the systematic sampling method. The primary outcome variable of the study was determined as "allergic rhinitis diagnosed by a specialist". Other variables included demographic information and the items of The European Community Respiratory Health Survey (ECRHS) Questionnaire. To estimate an expected prevalence of $20 \%(\mathrm{p}=0.2, \mathrm{q}=0.8)$ with a $95 \%$ confidence interval $(\mathrm{t}=1.96)$ with an accepted sample error (d) of 0.02 , the required sample size was calculated as a minimum of 1488 students.

\section{Statistical Analysis}

$\mathrm{R}$ version 4.0.2 was used for statistical analysis. Data were presented as frequency and percentage. Chi-Squared Test of Independence was used to analyze the relationship between allergic rhinitis diagnosis and other variables. Statistically significant variables were further analyzed with multivariate logistic regression. A p-value $<0.05$ was considered sufficient for statistical significance.

\section{Results}

Data was collected from a total of 2020 participants but 1714 were eligible for multivariate analysis. The mean age of the participants was $20.71 \pm 3.12$ years; $42.88(n=735)$ were male, and 57.12\% $(n=979)$ were female.

While the rate of those who thought that they had nasal allergies was $23.862 \%(n=409)$, the rate of those diagnosed by a doctor was $15.986 \%(n=274)$ (Table 1$)$.

The most common allergic symptom in the participants was sneezing, and the most common triggering factor was house dust (Table 2).

Participants' gender, skin allergy status, asthma status, parents' education level, parents' allergy status, sibling's allergy status, having a pet or not, house type and the residential area for the first two years of life were statistically significant when allergic rhinitis diagnosed by a specialist was taken into account (Table 3). However logistic regression results showed that only participants' 
Table I Allergic Rhinitis, Comorbidities and History

\begin{tabular}{|l|l|l|}
\hline & $\begin{array}{l}\text { No - Number } \\
\text { (\%) }\end{array}$ & $\begin{array}{l}\text { Yes - Number } \\
\text { (\%) }\end{array}$ \\
\hline $\begin{array}{l}\text { Nasal Allergies } \\
\text { AR* Diagnosed by }\end{array}$ & $1305(76.138)$ & $409(23.862)$ \\
Specialist & $1440(84.014)$ & $274(15.986)$ \\
Eczema & $1348(78.646)$ & $366(21.354)$ \\
Asthma & $1588(92.649)$ & $126(7.351)$ \\
Medication for AR* & $1538(89.732)$ & $176(10.268)$ \\
Regular Exercise & $881(51.4)$ & $833(48.6)$ \\
Smoking & $1205(70.303)$ & $509(29.697)$ \\
Mother Smoking & $1322(77.13)$ & $392(22.87)$ \\
Father Smoking & $978(57.06)$ & $736(42.94)$ \\
Parents' Allergy & $1395(81.389)$ & $319(18.611)$ \\
Siblings' Allergy & $1402(81.797)$ & $312(18.203)$ \\
Current Pet Ownership & $1318(76.896)$ & $396(23.104)$ \\
\hline
\end{tabular}

Abbreviation: *AR, allergic rhinitis.

Table 2 The Symptoms and Aggravating Factors

\begin{tabular}{|l|l|}
\hline Symptoms & Number (\%) \\
\hline Nasal Congestion & $505(17.142)$ \\
Nose Itching & $389(13.204)$ \\
Runny Nose & $423(14.358)$ \\
Sneeze & $557(18.907)$ \\
Redness and Itching in the Eye & $331(11.236)$ \\
Itching on the Palate & $167(5.669)$ \\
No Symptoms & $574(19.484)$ \\
Spring & $393(20.342)$ \\
Summer & $160(8.282)$ \\
Autumn & $199(10.3)$ \\
Winter & $435(22.516)$ \\
Throughout the Year & $238(12.319)$ \\
No Aggrevating Season & $507(26.242)$ \\
House.dust & $570(28.232)$ \\
Mold & $83(4.111)$ \\
Pollen & $435(21.545)$ \\
Pet & $58(2.873)$ \\
Other Allergens & $102(5.052)$ \\
No Aggrevating Allergens & $771(38.187)$ \\
Tomato & $4 I(2.356)$ \\
Peanuts & $15(0.862)$ \\
Strawberry & $22(1.264)$ \\
Chocolate & $32(1.839)$ \\
Egg & $34(1.954)$ \\
Milk & $22(1.264)$ \\
Other Foods & $98(5.632)$ \\
No Aggrevating Foods & $1476(84.828)$ \\
\hline
\end{tabular}

gender, asthma status, parents allergy status and having a pet or not were statistically significant when compared with having a doctor diagnosed AR or not. Females were
$42.8 \%$ more likely to have allergic rhinitis diagnosed by a specialist compared to males. Participants with asthma were $770.6 \%$ more likely to have a doctor confirmed allergy compared to participants without asthma. Participants with allergic parents were $114.3 \%$ were more likely to have allergic rhinitis diagnosed by a specialist to participants with non-allergic parents. Participants with a pet were $43.2 \%$ more likely to have a doctor confirmed allergy compared to participants without a pet (Table 4).

\section{Discussion}

The prevalence of allergic rhinitis is higher in males in childhood however from the age of fifteen onwards females are affected more. ${ }^{25,26}$ We surveyed university students with a mean age of $20.71 \pm 3.12$ years. The difference between males and females was scientifically significant in the chi-square test $(p=0.001)$. The female students were at a clear disadvantage in terms of suffering from allergic rhinitis as the regression test revealed a $42.8 \%$ more likelihood of suffering from AR compared to male students $(\mathrm{p}=0.021)$.

IgE-mediated allergy is hypothesized to be a systemic disease. ${ }^{27-29}$ This hypothesis is supported with the systemic presentation of the disease and comorbidities. A study by Pinart et $\mathrm{al}^{30}$ showed eczema (atopic dermatitis), allergic rhinitis, and asthma coexisted with a high percentage of relative risk in children. We found a scientifically significant relationship between both eczema-AR $(\mathrm{p}=0.006)$, and asthma-AR $(\mathrm{p}<0.001)$ in our chi-square test results which are in accordance with the literature. However, when the regression test was applied only asthma and AR had a scientifically significant correlation $(\mathrm{p}<0.001)$. The participants with an asthma diagnosis were seven times more likely to suffer from AR compared to those who did not have asthma. A 2017 study further examined the shared genetic origin of allergic rhinitis, asthma and atopic dermatitis. ${ }^{31}$ The risk of suffering from allergic rhinitis in university students increases significantly if either the parents $(p<0.001)$ or the siblings $(\mathrm{p}=0.001)$ have allergic rhinitis according to our chi-square test. However, the regression test only revealed a positive relationship between allergy in the parents and participants AR $(\mathrm{p}<0.001)$. The students were $114.3 \%$ more likely to have AR if their parents had any allergies. A study conducted with over 3000 students in Japanese university students also revealed a positive relationship between family history and AR but an 


\begin{tabular}{|c|c|c|c|c|c|c|c|c|c|c|}
\hline $\begin{array}{l}\frac{0}{2} \\
\frac{2}{N} \\
\stackrel{1}{0}\end{array}$ & $\begin{array}{l}\bar{o} \\
0\end{array}$ & $\begin{array}{l}\circ \\
\stackrel{0}{0} \\
0\end{array}$ & $\begin{array}{l}\overline{0} \\
\dot{v} \\
\text { val }\end{array}$ & on & $\begin{array}{l}\stackrel{L}{0} \\
\text { ô } \\
0\end{array}$ & 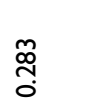 & 菅 & $\begin{array}{l}\bar{o} \\
\dot{v} \\
\dot{v}\end{array}$ & $\begin{array}{l}\overline{0} \\
\dot{v} \\
\text { val }\end{array}$ & थ્م \\
\hline 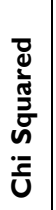 & $\begin{array}{l}\underset{0}{0} \\
\stackrel{\underline{=}}{=}\end{array}$ & 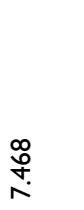 & 离 & $\stackrel{\infty}{0}$ & i & $\stackrel{n}{\underline{L}}$ & 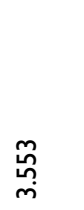 & $\frac{o}{\sigma}$ & $\underset{\substack{\infty \\
\infty}}{\stackrel{\infty}{\infty}}$ & 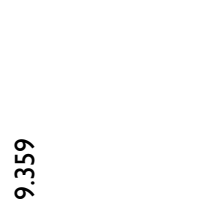 \\
\hline 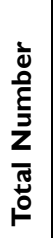 & 岀呑 & 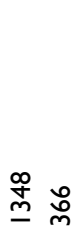 & 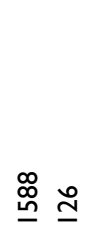 & $\bar{\infty} \underset{\infty}{\infty}$ & 岕 & ్ㅗ & $\stackrel{\infty}{\sigma} \stackrel{\circ}{\kappa}$ & œ & 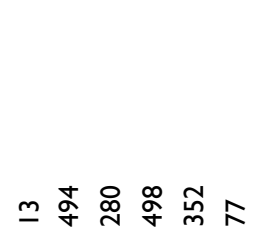 & to 음 $\frac{\infty}{n} q=$ \\
\hline 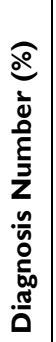 & 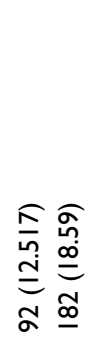 & 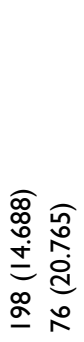 & 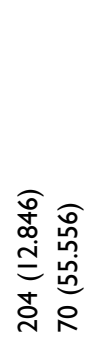 & 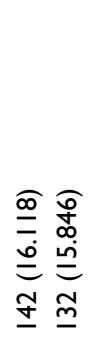 & 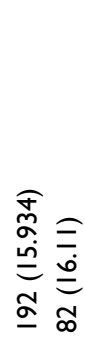 & 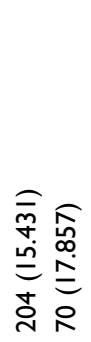 & 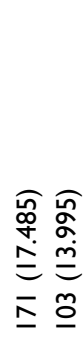 & 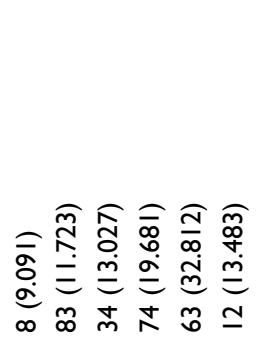 & 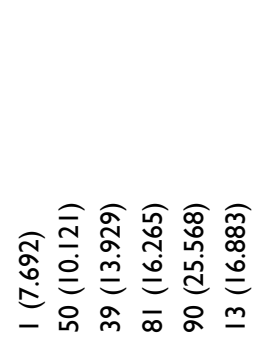 & 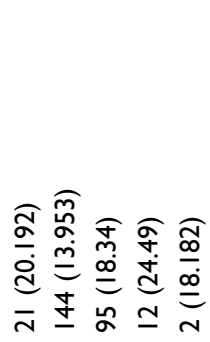 \\
\hline 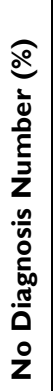 & 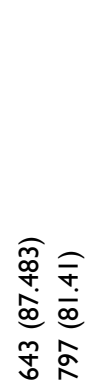 & 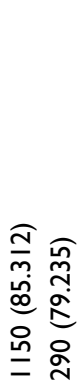 & 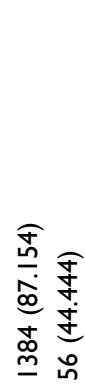 & 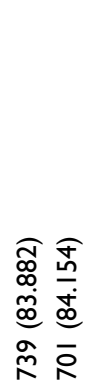 & 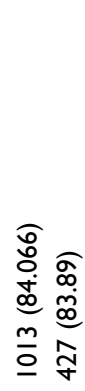 & 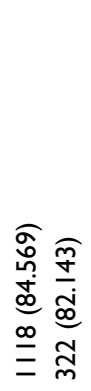 & 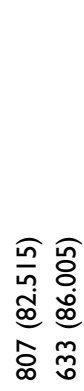 & 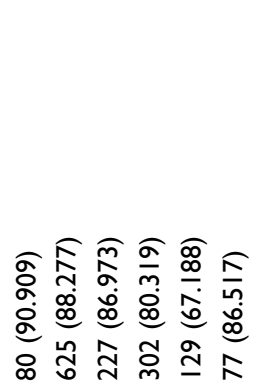 & 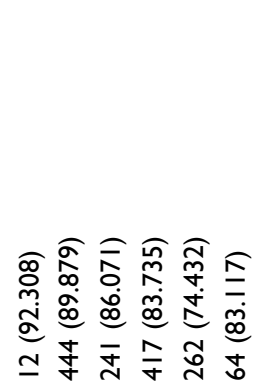 & 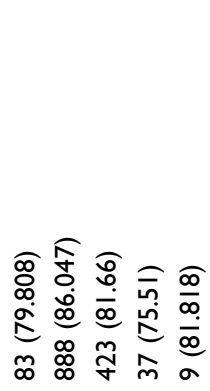 \\
\hline & $\frac{\frac{0}{\pi}}{\Sigma} \frac{\frac{0}{\pi}}{\frac{\pi}{\Psi}}$ & $20 \stackrel{0}{x}$ & $20 \stackrel{y}{x}$ & $\stackrel{0}{z} \overbrace{}^{y}$ & 20 & $2 \stackrel{0}{x}$ & 20 ֻั & 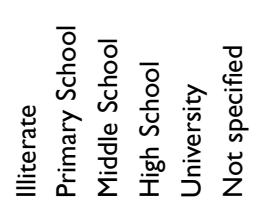 & 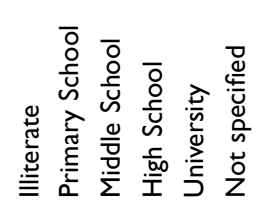 & 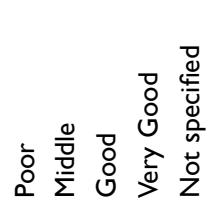 \\
\hline & 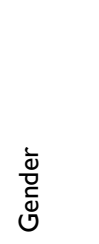 & 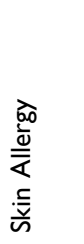 & 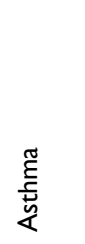 & 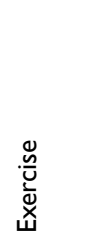 & 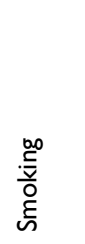 & 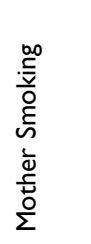 & 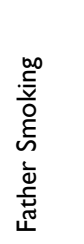 & 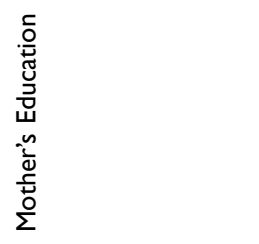 & 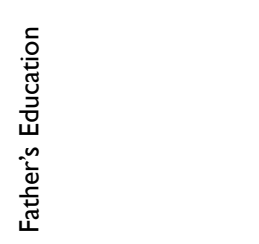 & 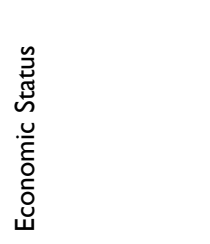 \\
\hline
\end{tabular}




\begin{tabular}{|c|c|c|c|c|c|c|c|}
\hline $\begin{array}{l}\bar{o} \\
\dot{0} \\
\dot{0}\end{array}$ & $\begin{array}{l}\overline{0} \\
\dot{0}\end{array}$ & $\begin{array}{l}\bar{\delta} \\
\dot{0}\end{array}$ & $\stackrel{\substack{\infty \\
\tilde{O}}}{o}$ & $\frac{\circ}{\circ}$ & $\begin{array}{l}\text { ò } \\
0\end{array}$ & 号 & $\overline{0}$ \\
\hline $\begin{array}{l}\widetilde{o} \\
+ \\
\sigma \\
\sigma\end{array}$ & $\stackrel{\stackrel{\text { }}{\text { I }}}{=}$ & 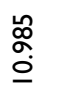 & $\frac{a^{2}}{2}$ & స్త్రి & 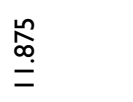 & $\frac{\sigma}{n}$ & సু \\
\hline$\stackrel{n}{\stackrel{n}{m}} \frac{a}{m}$ & $\frac{d}{d} \frac{m}{m}$ & $\frac{\infty}{m} \stackrel{\circ}{m}$ & 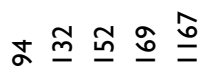 & సे $\pm \overline{\bar{\infty}}$ & 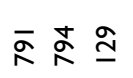 & స్ & fீ \\
\hline 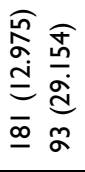 & 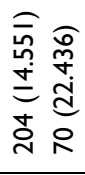 & 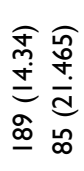 & 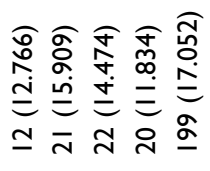 & 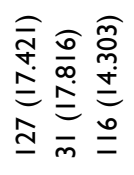 & 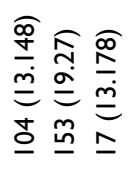 & 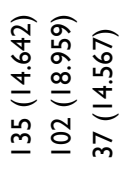 & 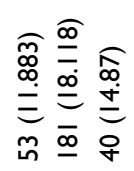 \\
\hline 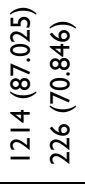 & 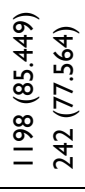 & 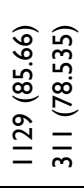 & 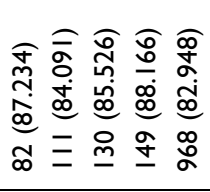 & 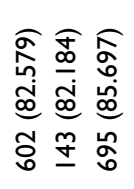 & 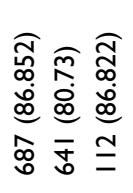 & 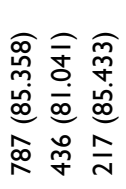 & 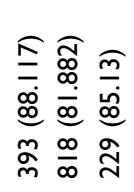 \\
\hline 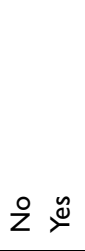 & $\stackrel{0}{z} \stackrel{y}{z}$ & zo vo & 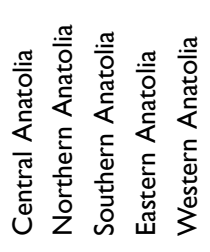 & 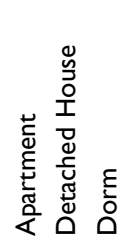 & 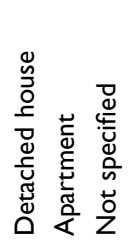 & 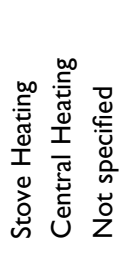 & 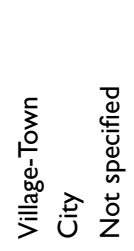 \\
\hline 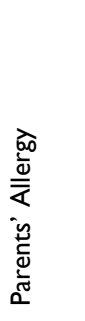 & 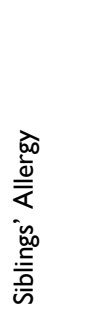 & 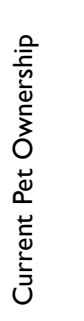 & 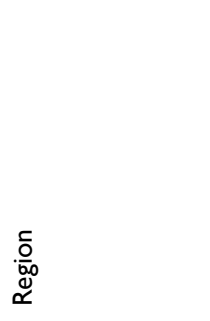 & 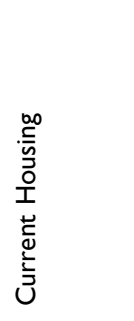 & 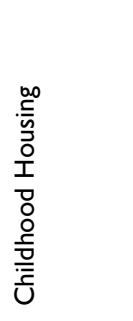 & 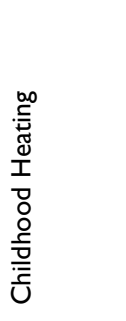 & 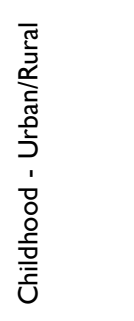 \\
\hline
\end{tabular}


Table 4 The Results of the Logistic Regression Test

\begin{tabular}{|c|c|c|c|c|c|}
\hline & & Estimate & $\mathrm{e}^{\wedge}$ Estimate & Std Error & p-value \\
\hline & Intercept & -3.448 & 0.032 & 1.133 & 0.002 \\
\hline Gender & Female & 0.356 & 1.428 & 0.154 & 0.021 \\
\hline Skin Allergy & Yes & 0.257 & 1.293 & 0.166 & 0.122 \\
\hline Asthma & Yes & 2.164 & 8.706 & 0.209 & $<0.001$ \\
\hline \multirow[t]{5}{*}{ Mother's Education } & Primary School & 0.014 & 1.014 & 0.417 & 0.972 \\
\hline & Middle School & -0.073 & 0.93 & 0.454 & 0.872 \\
\hline & High School & 0.356 & 1.428 & $0.44 I$ & 0.419 \\
\hline & University & 0.817 & 2.264 & 0.468 & $0.08 I$ \\
\hline & Not specified & -0.416 & 0.66 & 0.706 & 0.556 \\
\hline \multirow[t]{5}{*}{ Father's Education } & Primary School & 0.192 & 1.212 & 1.136 & 0.866 \\
\hline & Middle School & 0.524 & 1.689 & 1.142 & 0.646 \\
\hline & High School & 0.543 & 1.721 & 1.139 & 0.633 \\
\hline & University & 0.665 & 1.944 & 1.147 & 0.562 \\
\hline & Not specified & 1.426 & 4.162 & 1.26 & 0.258 \\
\hline Parents' Allergy & Yes & 0.762 & 2.143 & 0.165 & $<0.001$ \\
\hline Siblings' Allergy & Yes & 0.29 & 1.336 & 0.176 & 0.1 \\
\hline Current Pet Ownership & Yes & 0.359 & 1.432 & 0.16 & 0.025 \\
\hline \multirow[t]{2}{*}{ Childhood Housing } & Apartment & 0.223 & 1.25 & 0.181 & 0.22 \\
\hline & Not specified & 0.157 & 1.17 & 0.315 & 0.619 \\
\hline \multirow[t]{2}{*}{ Childhood - Urban/Rural } & City & 0.116 & 1.123 & 0.214 & 0.587 \\
\hline & Not specified & 0.08 & 1.083 & 0.247 & 0.746 \\
\hline
\end{tabular}

Note: Bolded figures indicate a scientifically significant relationship.

interesting finding in their study was if there was a large number of people living in the same household during preschool age it decreased the risk of AR. ${ }^{32}$

There is a distinct form of physical allergy called exercise-induced anaphylaxis. ${ }^{33}$ But the mechanisms of exercise-induced anaphylaxis are usually through food hypersensitivity. ${ }^{34}$ On the other hand, regular exercise can reduce the symptoms of allergic rhinitis due to increased VO2-max. ${ }^{35}$ Also, obesity is known to increase asthma severity and regular exercise can help keep a healthy weight. ${ }^{36}$ However, it does not increase the prevalence of neither asthma nor AR. ${ }^{35,36}$ There was no significant difference in $\mathrm{AR}$ prevalence in students who exercised regularly and those who did not $(\mathrm{p}=0.93)$. Further questioning is needed to reveal more about the relationship between AR severity and exercise type/ frequency.

It is known that smoking causes serious airway diseases such as chronic obstructive pulmonary disease however, there is no indication that smoking increases AR prevalence. ${ }^{37}$ The reason behind that could be the relatively older age of starting to smoke. We found no scientifically significant difference between students who smoked cigarettes and those who did not $(\mathrm{p}=0.985)$.

Effects of maternal smoking were established as early as the 1980s; it drastically increases cord serum $\operatorname{IgE}$ and IgD levels and considered to elevate the risk of developing an atopic disease. ${ }^{38}$

We did not question the maternal smoking in university students to prevent inaccurate data. However, we questioned whether their parents smoked or not and interestingly there was no significant difference (mother smoking, $\mathrm{p}=0.283$ /father smoking, $\mathrm{p}=0.059$ ). A seven-year study published in 1993 suggests since the discovery of the relationship between smoking and aggravated asthma the parents were purposely informed not to expose their children to smoke. Reducing the exposure to smoke then resulted in better quality of life in children who suffer 
from asthma. ${ }^{39}$ Since 1993, there has been various studies and campaigns against maternal smoking and smoke exposure of children. This could explain why we found no correlation between smoking parents and allergies in university students.

The environment plays a significant role in presentation of AR, eczema, and asthma and many other allergy diseases. ${ }^{40}$ The risk of having AR is four times higher in children who live in urban areas compared to those who live in rural areas and the risk of having asthma is almost eight-fold. $^{41}$ Traffic related air pollution was found to be a major factor in this. ${ }^{42}$ Supporting the importance of early childhood environment, we have found a statistically significant relationship between the type of house, area (rural/ urban) students lived in their early ages and AR prevalence $(\mathrm{p}=0.01)$

The type of house that lived in early childhood was also found to be significant $(\mathrm{p}=0.003)$. Once again there was no scientifically significant effect of the environment in the prevalence in older ages since no significant relationship was present between AR prevalence and the type of housing students lived currently $(p=0.196)$.

The parents' levels of education were found to be significant $(p<0.001)$. This is thought to be incidental since the level of education effects the type of house and the area (rural/urban) lived in. ${ }^{43,44}$ Although the economic status of students' families also affects the house and the area lived in it was not found to be statistically significant $(p=0.053)$. Which might be caused by the importance of agriculture in the economy of Anatolia region.

The relationship between having a pet and AR is a controversial issue. Many studies showed a reduced AR prevalence in populations who had pets in their homes in their early life. ${ }^{45,46}$ However, Luo et $\mathrm{al}^{47}$ found a positive correlation between pet ownership and AR despite most of the pet owners being in rural areas where the AR prevalence is established to be almost four times less. ${ }^{48}$ We questioned the current ownership of pets which was found to be positively related to $A R(p=0.001)$ in the chi-squared test and the regression test $(p<0.025)$.

The majority of our participants were from Western Anatolia (68.1\%) which may be considered as a limitation but considering that over $43.5 \%$ of the population of Turkey is located in the Western Anatolia region the distribution is expected. Besides, no significant relationship was found between where the students were from and AR prevalence despite relatively even distribution $(p=0.387)$. The major cause of this is considered that in all areas of
Anatolia the most common allergens are house dust (D. farinae) and pollen groups. ${ }^{48-50}$

In conclusion, this study investigated the university students from Anatolia whose confirmed diagnosis of AR prevalence was $15.986 \% \%$. Female gender, family history, kind of house during the first years of life, economic status, etc., were associated risk factors in the chi-square test that shows AR has both genetic and environmental etiologies. Further analysis using regression test also supported the idea of AR having both genetic and environmental etiologies with female gender, parents' history, having asthma and current pet ownership being scientifically significant in relationship to AR. Meanwhile, smoking and regular exercise were neutral factors for AR prevalence. This study creates a starting point for understanding AR prevalence and epidemiological factors in university students from Anatolia. Considering that $23.862 \%$ of the participants thought they had allergies but the rate of those diagnosed by a clinician was 15.986\% further investigation and tests (eg, allergic prick test) should be performed in the future.

\section{Data Sharing Statement}

The statistical data used to support the findings of this study are available from the author upon request.

\section{Acknowledgments}

The authors thank the surveyor and participant students for their cooperation in the data collection. Special thanks are due to Alperen Elibol for his support in the statistical analysis.

\section{Funding}

This study was not funded by any organization.

\section{Disclosure}

The authors declared no potential conflicts of interest with respect to this work, the research, authorship, and/or publication of this article.

\section{References}

1. Özdemir Ö, Elmas B. Variable prevalence of allergic rhinitis and risk factors affecting the prevalence. Kulak Burun Bogaz Ihtis Derg KBB. 2016;26(6):371-382.

2. Okubo K, Kurono Y, Ichimura K, et al. Japanese guidelines for allergic rhinitis 2020. Allergol Int. 2020;69(3):331-345. doi:10.1016/j. alit.2020.04.001

3. Sikorska-Szaflik H, Sozańska B. The role of vitamin D in respiratory allergies prevention. why the effect is so difficult to disentangle? Nutrients. 2020;12(6):1801-1810. doi:10.3390/nu12061801 
4. Small P, Keith PK, Kim H. Allergic rhinitis. Allergy, asthma. Clin Immunol off J Can Soc Allergy Clin Immunol. 2018;14(Supp1 2):51-55.

5. Wise SK, Lin SY, Toskala E, et al. International consensus statement on allergy and rhinology: allergic rhinitis. Int Forum Allergy Rhinol. 2018;8(2):108-352.

6. Bauchau V, Durham SR. Prevalence and rate of diagnosis of allergic rhinitis in Europe. Eur Respir J. 2004;24(5):758-764. doi:10.1183/ 09031936.04.00013904

7. Sapsaprang S, Setabutr D, Kulalert P, Temboonnark P, Poachanukoon O. Evaluating the impact of allergic rhinitis on quality of life among Thai students. Int Forum Allergy Rhinol. 2015;5 (9):801-807. doi:10.1002/alr.21540

8. Janson C, Anto J, Burney P, et al. The european community respiratory health survey: what are the main results so far? European community respiratory health survey ii. Eur Respir J. 2001;18 (3):598-606. doi:10.1183/09031936.01.00205801

9. Hossenbaccus L, Linton S, Garvey S, et al. Towards definitive management of allergic rhinitis: best use of new and established therapies. Allergy, asthma. Clin Immunol off J Can Soc Allergy Clin Immunol. 2020;16(39):1-17.

10. An S-Y, Choi HG, Kim SW, et al. Analysis of various risk factors predisposing subjects to allergic rhinitis. Asian Pacific $J$ Allerg Immunol. 2015;33(2):143-151.

11. Alsowaidi S, Abdulle A, Shehab A, et al. Allergic rhinitis: prevalence and possible risk factors in a Gulf Arab population. Allergy. 2010;65 (2):208-212. doi:10.1111/j.1398-9995.2009.02123.x

12. Cingi C, Cakli H, Us T, et al. The prevalence of allergic rhinitis in urban and rural areas of Eskişehir-Turkey. Allergol Immunopathol. 2005;33(3):151-156. doi:10.1157/13075698

13. Özdemir N, Ucgun I, Metintas S, Kolsuz M, Metintas M. The prevalence of asthma and allergy among university freshmen in Eskisehir, Turkey. Respir Med. 2000;94(6):536-541. doi:10.1053/ rmed.1999.0728

14. Topal Ö, Erbek SS, Erbek S, Çakmak Ö. Konya yöresinde yaşayan perennial alerjik rinitli hastalarda epidemiyolojik özellikler, alerjen dağılımı ve semptom ciddiyeti [Epidemiological characteristics, allergen distribution and symptom severity in patients with perennial allergic rhinitis living in Konya region]. Kulak Burun Bogaz Ihtis Derg. 2008;18(4):227-231.

15. Topuz B, Kara CO, Ardiç N, et al. The prevalence of allergic rhinitis in the adult urban population of Denizli. Kulak Burun Bogaz Ihtis Derg. 2005;14(5-6):106-109.

16. Jones NS, Smith PA, Carney AS, Davis A. The prevalence of allergic rhinitis and nasal symptoms in Nottingham. Clin Otolaryngol Allied Sci. 1998;23(6):547-554. doi:10.1046/j.1365-2273.1998.2360547.x

17. Didier A, Chanal I, Klossek JM, Mathieu J, Bousquet J. La rhinite allergique: le point de vue du patient [Allergic rhinitis: the patient's perspective]. Rev Fr d'allergologie d'immunologie Clin. 1999;39 (3):171-185. doi:10.1016/S0335-7457(99)80002-1

18. Bousquet J, Bullinger M, Fayol C, Marquis P, Valentin B, Burtin B. Assessment of quality of life in patients with perennial allergic rhinitis with the French version of the SF-36 Health Status Questionnaire. J Allergy Clin Immunol. 1994;94(2):182-188. doi:10.1053/ai.1994.v94.a54939

19. Cockburn IM, Bailit HL, Berndt ER, Finkelstein SN. Loss of work productivity due to illness and medical treatment. J Occup Environ Med. 1999;41(11):948-953. doi:10.1097/00043764-19991100000005

20. Simons FER. Learning impairment and allergic rhinitis in allergy and asthma proceedings. OceanSide Publ. 1996;17(4):185-189.

21. Seedat RY, Sujee M, Ismail W, et al. Allergic rhinitis in medical students at the University of the Free State. South Afri Fam Pract. 2018;60(4):121-125. doi:10.1080/20786190.2018.1437869

22. Skoner DP. Complications of allergic rhinitis. J Allergy Clin Immunol. 2000;105(6):605-609. doi:10.1067/mai.2000.106150
23. Sunyer J, Anto JM, Tobias A, Burney P. Generational increase of self-reported first attack of asthma in fifteen industrialized countries. Eur Respir J. 1999;14(4):885-891.

24. von Elm E, Altman DG, Egger M, et al. The strengthening the reporting of observational studies in epidemiology (strobe) statement: guidelines for reporting observational studies. $J$ Clin Epidemiol. 2008;61(4):344-349. doi:10.1016/j.jclinepi.2007.11.008

25. Ford ES, Mannino DM, Homa DM, et al. Self-reported asthma and health-related quality of life: findings from the behavioral risk factor surveillance system. Chest. 2003;123(1):119-127. doi:10.1378/ chest.123.1.119

26. Jensen-Jarolim E, Untersmayr E. Gender-medicine aspects in allergology. Allergy. 2008;63(5):610-615. doi:10.1111/j.13989995.2008.01645.x

27. Tollefsen E, Langhammer A, Bjermer L, Romundstad P, Holmen TL. Allergy: a systemic disease? The HUNT and Young-HUNT study, Norway. Pediatr Allerg Immunol. 2008;19(8):730-736. doi:10.1111/ j.1399-3038.2008.00732.x

28. Pucci S, Incorvaia C. Allergy as an organ and a systemic disease. Clin Exp Immunol. 2008;118(1):112-119.

29. Sin E, Prachi A, Marianne F. A link: allergic rhinitis, asthma; systemic lupus erythematosus. Autoimmun Rev. 2016;15(5):487-491. doi:10.1016/j.autrev.2016.02.003

30. Pinart M, Benet M, Annesi-Maesano I, et al. Comorbidity of eczema, rhinitis, and asthma in ige-sensitised and non-ige-sensitised children in medall: a population-based cohort study. Lancet Respir Med. 2014;2(2):131-140. doi:10.1016/S2213-2600(13)70277-7

31. Ferreira M, Vonk J, Baurecht $\mathrm{H}$, et al. Shared genetic origin of asthma, hay fever and eczema elucidate allergic disease biology. Nat Genet. 2017;49(12):1752-1757. doi:10.1038/ng.3985

32. Nishijima H, Suzuki S, Kondo K, Yamasoba T, Yanagimoto S. Environmental factors associated with allergic rhinitis symptoms in Japanese university students: a cross-sectional study. Auris Nasus Larynx. 2018;45(5):1006-1013. doi:10.1016/j.anl.2018.02.004

33. Albert L, Nicholas A, McFadden S, et al. Exercise-induced anaphylaxis: a distinct form of physical allergy. $J$ Allerg Clin Immunol. 1983;71(3):311-316. doi:10.1016/0091-6749(83)90085-4

34. Maulitz R, Pratt DS, Schocket AL, et al. Exercise-induced anaphylactic reaction to shellfish. J Allerg Clin Immunol. 1979;63 (6):433-434. doi:10.1016/0091-6749(79)90218-5

35. Toennesen L, Soerensen D, Hostrup M, et al. Feasibility of high-intensity training in asthma. Eur Clin Respir J. 2018;5 (1):1468-1476. doi:10.1080/20018525.2018.1468714

36. Bergeron C, Louis-Philippe B, Qutayba H. Obesity, allergy and immunology. J Allerg Clin Immunol. 2005;115(5):1102-1104. doi:10.1016/j.jaci.2005.03.018

37. Nagasaki $T$, Matsumoto $H$. Influences of smoking and aging on allergic airway inflammation in asthma. Allergol Int. 2013;62 (2):171-179. doi:10.2332/allergolint.12-RA-0523

38. Magnusson C. Maternal smoking influences cord serum $\operatorname{IgE}$ and $\operatorname{IgD}$ levels and increases the risk for subsequent infant allergy. $J$ Allerg Clin Immunol. 1986;78(5):898-904. doi:10.1016/0091-6749(86) 90237-X

39. Murray A, Morrison B. The decrease in severity of asthma in children of parents who smoke since the parents have been exposing them to less cigarette smoke. J Allerg Clin Immunol. 1993;91 (1):102-110. doi:10.1016/0091-6749(93)90302-V

40. Burbank AJ, Sood AK, Kesic MJ, Peden DB, Hernandez ML. Environmental determinants of allergy and asthma in early life. $J$ Allergy Clin Immunol. 2017;140(1):1-12. doi:10.1016/j. jaci.2017.05.010

41. Majkowska-Wojciechowska B, Pelka J, Korzon L, et al. Prevalence of allergy, patterns of allergic sensitization and allergy risk factors in rural and urban children. Wiley Online Library. 2007;62 (5):1044-1050. 
42. Qihong D, Lu C, Li Y, et al. Exposure to outdoor air pollution during trimesters of pregnancy and childhood asthma, allergic rhinitis, and eczema. Environ Res. 2016;150(3):119-127. doi:10.1016/j. envres.2016.05.050

43. Sicular T, Yue X, Björn G, et al. The urban \& rural income gap and inequality in China. Rev Income Wealth. 2007;53(1):93-126. doi:10.1111/j.1475-4991.2007.00219.x

44. Çiftçi C, Kangallı S. Eğitim ve gelir [Education and income]. Ege Akademik Bakis. 2015;15(1):141-152.

45. Gennaro L, D'Amatoa G, Luciana D, et al. The effect of pet ownership on the risk of allergic sensitisation and bronchial asthma. Respir Med. 2005;99(2):227-233. doi:10.1016/j.rmed.2004.05.012

46. Hesselmar B, Hicke-Roberts A, Lundell AC, et al. Pet-keeping in early life reduces the risk of allergy in a dose-dependent fashion. PLoS One. 2018;13(12):1-13. doi:10.1371/journal.pone.0208472
47. Luo S, Sun Y, Hou J, et al. Pet keeping in childhood and asthma and allergy among children in Tianjin area, China. PLoS One. 2018;13 (5):1-9.

48. Özdemir Ö. Variable prevalence of allergic rhinitis and risk factors affecting the prevalence. Turk J Ear Nose Throat. 2016;26 (6):371-382. doi:10.5606/kbbihtisas.2016.97059

49. Aktaş A, Rahman S, Elbi H. Üniversite öğrencilerinde allerjik rinit sıklığı ve ilişkili faktörler. [Frequency of allergic rhinitis in university students and related factors]. Celal Bayar Üniversitesi Saglik Bilimleri Enstitüsü Dergisi. 2015;2(2):36-40.

50. Ayçin D, Muharrem B, Kenan Ç. Prick test results in patients with allergic rhinitis and asthma. J Health Sci Med. 2020;3(3):245-249. doi:10.32322/jhsm. 710017

\section{Publish your work in this journal}

The Journal of Asthma and Allergy is an international, peer-reviewed open-access journal publishing original research, reports, editorials and commentaries on the following topics: Asthma; Pulmonary physiology; Asthma related clinical health; Clinical immunology and the immunological basis of disease; Pharmacological interventions and new therapies. The manuscript management system is completely online and includes a very quick and fair peer-review system, which is all easy to use. Visit http://www.dovepress.com/testimonials.php to read real quotes from published authors. 